\title{
Hipersensibilidade dentinária cervical: uma revisão da literatura
}

\author{
Cervical dentinal hypersensitivity: a review of literature
}

Micheline Sandini Trentin*

Juliane Bervian**

\section{Resumo}

Introdução: a hipersensibilidade dentinária cervical (HSDC) é caracterizada pela perda de esmalte e de cemento na região cervical dos dentes e consequente exposição dos túbulos dentinários que levam a uma condição dolorosa e de desconforto para o paciente. Objetivo: este estudo tem como objetivo abordar os fatores etiológicos, a prevalência e as diversas formas de tratamento relacionadas à hipersensibilidade dentinária cervical. Revisão de literatura: sendo relatada com uma prevalência em torno de $30 \%$ da população, essa perda de estrutura dentária ocorre em virtude da formação de lesões cervicais por processos de recessão gengival, abrasão, erosão, abfração ou pela associação de dois ou mais fatores. O conhecimento da etiologia de qualquer doença ou condição bucal é primordial para um tratamento seguro e efetivo, portanto, esta revisão da literatura tem por finalidade informar a etiologia, a prevalência e as opções de tratamento para a hipersensibilidade dentinária, por meio de uma revisão em bases de dados nacionais e internacionais (pubmed, scielo, bireme, cochrane). Alguns tratamentos clínicos, tanto de uso doméstico, como profissional apresentaram-se efetivos: aplicação de oxalato de potássio, cloreto de estrôncio, vernizes fluorados, fluoreto de sódio, laser de baixa potência, dentifrícios dessensibilizantes, sistemas adesivos e procedimentos restauradores. Considerações finais: portanto, a identificação e a remoção dos fatores etiológicos são essenciais para o sucesso do tratamento da HSDC, quando associadas à obliteração dos túbulos dentinários, tendo como resultado a efetiva redução do movimento do fluido dentro dos túbulos e a diminuição da dor.

Palavras-chave: Diagnóstico. Sensibilidade da dentina. Prevenção e controle. Hidrodinâmica.

\section{Introdução}

A hipersensibilidade dentinária cervical é um fenômeno sintomático relacionado à clínica odontológica que causa grande desconforto para o paciente e é de difícil solução para o cirurgião-dentista. Também poderia ser definida como superfícies dentinárias expostas a estímulos térmicos, evaporativos, tácteis, mecânicos ou osmóticos ocorridos na região cervical dos dentes, e que não pode ser atribuída a nenhuma forma de defeito ou patologia dental ${ }^{1,2}$.

Para ocorrer a condição de HSDC é necessário que alguma porção da dentina esteja exposta ao ambiente bucal, ou seja, o esmalte e o cemento que recobre a dentina tenham sido perdidos ou removidos. A ausência de cemento na região radicular permite que os túbulos dentinários se estendam perifericamente causando dor. Com isso, é frequente o paciente procurar o cirurgião-dentista, relatando dor ao ingerir alimentos frios, no ato de respirar ou de escovar os dentes, acreditando que esse desconforto tenha origem de um processo carioso ${ }^{3}$.

A causa da hipersensibilidade dentinária está diretamente relacionada aos túbulos dentinários abertos, presentes em toda a extensão da dentina. Essa exposição dentinária ocorre frequentemente como resultado da recessão gengival associada à exposição das superfícies radiculares e à perda de esmalte ligada ao desgaste ou ao traumatismo dental. $\mathrm{O}$ desgaste dentário refere-se à perda irreversível de estrutura dentária e inclui processos de recessão gengival, abrasão, erosão, abfração ou associação de 
dois ou mais fatores. Estas lesões são denominadas lesões cervicais ${ }^{4,5}$.

Para a perda de estrutura dentária alguns dos tratamentos apresentados não são eficazes, porém há terapias efetivas como: aplicação de oxalato de potássio, de cloreto de estrôncio, de vernizes fluorados, de fluoreto de sódio, de laser, de dentifrícios dessensibilizantes, de sistemas adesivos e de procedimentos restauradores ${ }^{6,7}$. Portanto, a identificação e a remoção dos fatores etiológicos são essenciais ao sucesso do tratamento da HSDC, quando associadas à obliteração dos túbulos dentinários, tendo-se como resultado a efetiva redução do movimento do fluído dentro desses e a diminuição da dor ${ }^{2,8}$.

Assim, este estudo tem como objetivo abordar os fatores etiológicos, a prevalência e as diversas formas de tratamento relacionado à hipersensibilidade dentinária cervical.

\section{Revisão da literatura}

A hipersensibilidade dentinária cervical ocorre muitas vezes na forma de dor, durante hábitos corriqueiros do portador, tais como alimentação, diferenças de temperatura nos líquidos ingeridos, escovação dental e até muitas vezes, no simples ato de falar, devido à passagem do ar pela cavidade bu$\mathrm{cal}^{9}$. A HSDC caracteriza-se como uma dor aguda e passageira, resultante das superfícies dentinárias expostas a estímulos térmicos, evaporativos, mecânicos ou osmóticos ocorridos na região cervical dos dentes, e que não pode ser atribuída a nenhuma forma de defeito ou patologia dental ${ }^{2}$.

O termo hiperestesia dentinária pode ser utilizado para diferenciar entre uma situação de extrema sensibilidade a um determinado estímulo, e a dor resultante de eventos de natureza patológica ${ }^{10}$.

A prevalência relatada na literatura é de que a hipersensibilidade dentinária atinge de 25 a $30 \%$ da população. A maioria dos pacientes possui de 26 a 35 anos de idade e $53,8 \%$ dos dentes atingidos são pré-molares e $87 \%$ dos casos atingem a face vestibular dos dentes ${ }^{3}$. Liu et al. ${ }^{11}$ (1998) mostraram $32 \%$ e Palma et al. ${ }^{12}$ (2005) encontraram uma prevalência de $71,43 \%$ de indivíduos com HSDC.

Essa diferença de prevalência em parte é explicada pela diferença de populações incluídas nos estudos. Variações no diagnóstico e na terminologia, diferentes locais de estudo, diferenças alimentares e hábitos de higiene oral ${ }^{13,14}$ podem dificultar estudos epidemiológicos. Nota-se, no entanto, que a prevalência está aumentando e os profissionais estão cada vez mais cientes dessa condição ${ }^{2}$. É relevante também destacar que os dentes estão sendo mantidos na cavidade bucal com maior frequência, à medida que a população envelhece, a incidência da perda de superfície dentária não cariosa está aumentando e lesões cervicais não cariosas estão presentes numa variedade de formas ${ }^{14}$.

\section{Etiologia}

Os fatores etiológicos relacionados podem ser divididos em dois tipos de lesões: com perda de estrutura dental - que corresponde à erosão, à abrasão e à abfração -, e aquelas sem perda de estrutura dental, que são as exposições radiculares. Segundo Pereira $^{15}$ (2001), o grau de HSDC apresenta variação nos diferentes indivíduos, podendo, variar de dente para dente, no mesmo paciente, e até o mesmo dente apresentar diferentes graus de sensibilidade. A resposta à dor sofre variação de acordo com o estímulo aplicado, é recorrente e pode desaparecer sem tratamento.

Segundo Garcia et al. ${ }^{16}$ (1996), durante os procedimentos de raspagem e alisamento radicular indispensáveis ao tratamento da doença periodontal, ou até em pacientes que receberam tratamento periodontal cirúrgico, remove-se cemento e porções de dentina da superfície radicular, expõem-se os túbulos dentinários e, consequentemente, os prolongamentos de Tomes. Eventualmente, devido à retração gengival ocasionada pelo pós-operatório da raspagem, a superfície radicular e os túbulos dentinários abertos ficam expostos ao meio bucal e são submetidos a estímulos térmicos, osmóticos e elétricos, podendo conduzir uma hipersensibilidade dentinária, provocando dor ao paciente. Ao contrário do controle meticuloso de placa que diminui a sensibilidade dentinária radicular ${ }^{17}$.

A perda de esmalte dentário na ausência de recessão gengival pode envolver qualquer local do dente e é geralmente resultado da ação combinada da atrição, da abrasão e da erosão. A abrasão envolve a perda de esmalte através de mecanismos físicos que não envolvem o contato dente a dente, como nas áreas cervicais dos dentes. Resulta muitas vezes em lesões cervicais angulares, cuneiformes, nas superfícies vestibulares dos caninos maxilares e pré-molares e também nas superfícies linguais dos molares $^{18}$. Atribuiu-se ao desenvolvimento dessas lesões uma técnica excessiva e inadequada de escovação dos dentes. Quando a carga de oclusão excêntrica leva à flexão de cúspides, originando lesão nas linhas de esmalte na linha gengival, com consequente enfraquecimento da estrutura dentária, essas lesões são denominadas abfração e caracterizam-se pela presença de um entalhe cervical profundo em forma de V. Além disso, pode-se observar esse tipo de lesão cervical não cariosa, quando se realizam restaurações repetidamente na área cervical.

A erosão define-se como sendo a perda de tecido dentinário por dissolução química resultante de ácidos extrínsecos ou intrínsecos ${ }^{19}$. Como o ph crítico do esmalte dentário é de aproximadamente 5,5, qualquer solução com ph menor poderá causar erosão, especialmente se o ataque for de longa duração e repetir-se frequentemente. Por isso, o consumo de substâncias ácidas está associado ao diagnóstico de HSDC $^{12}$. Quando testado o potencial erosivo de 
sucos de fruta artificiais em pó, refrigerantes, isotônicos e chás enlatados, verificou-se que todas as bebidas apresentam significativo potencial erosivo in vitro, apresentando ph inferior a 3,72 , a $23{ }^{\circ} \mathrm{C}$ em temperatura ambiente ${ }^{20}$.

A exposição a ácidos extrínsecos é resultado de uma dieta rica em alimentos ácidos; enquanto os ácidos intrínsecos consistem em larga escala no ácido gástrico resultante da doença de refluxo gastroesofágico, distúrbios alimentares (anorexia e bulimia nervosa) ou dos efeitos secundários de medicamentos que irritam a mucosa gástrica ou causam náusea e vômito ${ }^{21}$. A saliva é um dos fatores biológicos de maior importância na proteção dos tecidos orais. A diminuição do fluxo salivar e a capacidade de tamponamento são duas das propriedades mais importantes nesse processo ${ }^{22}$.

Diversas proteínas salivares têm sido identificadas como componentes importantes da película dentária, sendo relevantes no desenvolvimento da erosão. Essas são as proteínas com alta afinidade ao cálcio que podem se depositar rapidamente a superfície do esmalte. Apesar de ser um fator importante de proteção, já foi demonstrado que esse efeito está limitado apenas à superfície do esmalte. Nenhuma, ou pouquíssima proteção contra a erosão tem sido observada para a dentina radicular ${ }^{6}$.

A teoria aceita para explicar a origem da dor da hipersensibilidade é a Teoria Hidrodinâmica, proposta por Brännström na década de 1960. Segundo essa teoria, quando os túbulos dentinários são expostos ao meio bucal e há estímulo sobre a superfície dentária, o fluido no interior dos túbulos se desloca. Esse deslocamento do fluido intratubular pode ativar receptores mecânicos nos nervos, estimular e distorcer as fibras nervosas presentes entre os odontoblastos, gerando a sensação dolorosa ${ }^{23,24}$.

\section{Tratamento da HSDC}

Antes do tratamento estabelecido é importante esclarecer que os termos sensibilidade e hipersensibilidade não são sinônimos. Sensibilidade dentinária é a resposta normal do indivíduo a um estímulo, enquanto hipersensibilidade denota uma sensibilidade excessiva estando relacionada à abrasão, à erosão, à atrição ou a perdas de estruturas periodontais.

A condição clínica da lesão é imprescindível para a escolha do tratamento, assim as áreas sensíveis sem perda aparente de estrutura, portanto sem cavitação irão receber um tipo de tratamento. Ao contrário das lesões que possuem perda de estrutura com formação de cavidade onde é possível executar uma restauração adequada ${ }^{3}$.

Independentemente do tipo de tratamento instituído, 20 a $40 \%$ dos dentes com hipersensibilidade dentinária normalmente apresentam melhora no período de quatro a oito semanas pós-tratamento ${ }^{10}$.

Em acordo com a Teoria Hidrodinâmica, Pashley ${ }^{24}$ (1994) sugeriu que seria possível diminuir a sensibilidade dentinária pela oclusão dos túbulos dentinários, evitando dessa forma, que o estímulo seja transmitido da dentina à polpa. Partindo de tal proposição, diversas substâncias têm sido testadas na tentativa de eliminar essa situação, como a utilização de dentifrícios à base de cloreto de estrôncio, aplicação de agentes fluoretados com ou sem auxílio de aparelhos de iontoforese, de pastas aquosas de hipofosfato de cálcio ou de hidróxido de cálcio, de jato de bicarbonato de sódio, vernizes, de laser de baixa intensidade, de selantes e de produtos à base de oxalato de potássio. Vários são os mecanismos de ação dos agentes dessensibilizantes que, de alguma forma aliviam a dor: desnaturação das terminações nervosas ou materiais orgânicos na extremidade dos odontoblastos, formação de dentina secundária no interior da polpa, inibição da inflamação no interior da polpa por meio de corticosteróides e oclusão dos túbulos dentinários por meios mecânicos ${ }^{8,16}$.

Os agentes dessensibilizantes são capazes de promover a oclusão dos túbulos dentinários com a finalidade de diminuir o deslocamento do fluido dentinário que é essencial para a transmissão do estímulo que provoca a reação dolorosa ${ }^{16}$. O componente dessensibilizante utilizado no adesivo Gluma é o glutaraldeído (GA), que é a substância ativa do Gluma Desensitizer ${ }^{\circledR}$ O glutaraldeído reage com a albumina sérica nos fluidos dentinários pelo processo de coagulação, interagindo, dessa forma, com o mecanismo hidrodinâmico da hipersensibilidade dentinária ${ }^{25,26}$.

Estudo in vitro foi realizado para testar o gel nitrato de potássio a $2 \%$ com fluoreto de sódio a $2 \%$ e o verniz com $5 \%$ de fluoreto de sódio na redução da permeabilidade dentinária, verificando a obliteração parcial dos túbulos dentinários. A utilização contínua dos agentes dessensibilizantes pode contribuir para uma maior efetividade desses na obliteração dos túbulos dentinários ${ }^{13}$. Em estudo realizado in vivo, a utilização do verniz fluoretado se mostrou superior para o tratamento de hipersensibilidade dentinária ao gel com nitrato de potássio e íons flúor ${ }^{27}$.

Para o tratamento da hipersensibilidade, verifica-se também a utilização do fluoreto de sódio que, em contato com a dentina reage com o cálcio ionizado do fluido tubular, formando um precipitado de fluoreto de cálcio insolúvel responsável pela diminuição da condução hidráulica nos túbulos dentinários. Os dentifrícios com cálcio apresentam resultados mais efetivos na obliteração dos túbulos dentinários $^{28}$. Contudo, observou-se que o efeito das soluções fluoretadas é de curta duração, além de serem necessárias várias aplicações para obtenção de um resultado significativo na redução da dor ${ }^{13,15}$.

Medidas preventivas indicam o emprego de técnicas adequadas de escovação associadas aos dentifrícios menos abrasivos ${ }^{6}$. Tanto a escovação dentária, quanto o dentifrício fluoretado parecem ser fatores relevantes influenciando a perda de superfície 
dentária. Ainda que se desconheça a existência de diferenças clínicas no emprego de escovas manuais e elétricas, parece razoável recomendar que pacientes com hipersensibilidade dentinária utilizem escovas elétricas com dentifrícios de baixa abrasividade ${ }^{7}$.

Os dentifrícios, com propriedades dessensibilizantes, reduzem significativamente a hipersensibilidade dentinária. Estudo clínico randomizado comprovou que em três dias os testes mostraram resultados positivos, comprovando a indicação desses produtos na prática clínica ${ }^{29}$.

A terapia com laser de baixa e de alta potência é utilizada no tratamento da hipersensibilidade dentinária, podendo serem observados, na literatura, resultados promissores com lasers Nd:YAG, Er:YAG, $\mathrm{CO}_{2}$ e diodo ${ }^{2}$. Os lasers de baixa potência têm ação sobre a transmissão nervosa, enquanto os de alta potência ocluem túbulos dentinários por fusão ${ }^{3}$. A utilização do diodo, embora seja promissora, causa receio em função de injúrias causadas ao tecido pulpar, o que pode comprometer sua vitalidade ${ }^{30}$.

Estudos verificaram que, após a segunda aplicação do raio laser $\mathrm{HeNe}$, a intensidade da dor apresentava-se entre nula e mínima na totalidade dos casos. A diminuição da sensibilidade também foi encontrada após a segunda semana de aplicação do laser Nd:YAG e o mecanismo de ação, embora ainda não tenha sido confirmado, é a obliteração dos túbulos dentinários. Essa obliteração também pode estar envolvida na dessensibilização dentinária após a irradiação do laser de baixa potência.

Em estudo in vivo foi verificado que o laser de AsGaAl e o laser de Nd:YAG são efetivos no tratamento de hipersensibilidade dentinária e não houve diferença significante entre os resultados obtidos entre o laser de AsGaAl e o laser de Nd:YAG. Ambos os lasers proporcionam uma diminuição significante na hipersensibilidade dentinária, porém o laser de baixa potência tem a vantagem de ser um aparelho mais acessível economicamente, de mais fácil manuseio, que oferece menor risco à integridade pulpar e, portanto, passível de uso na clínica diária ${ }^{31}$.

A utilização do laser na Odontologia, desde 1960, tem mostrado aumento na eficiência, na especificidade e, atualmente, apresenta maior comodidade e menor custo ${ }^{32}$. Estudos atuais estão associando com sucesso a utilização do laser com outros métodos na obliteração dos túbulos dentinários ${ }^{33}$. A associação de Nd: YAG e Gluma Desensitizer é uma estratégia de tratamento eficaz que tem efeitos imediatos e em longo prazo na redução da hipersensibilidade dentinária ${ }^{34}$.

Outros tipos de tratamento mais invasivos e menos conservadores como os citados acima também são propostos, como o reposicionamento coronal de retalhos periodontais para cobrir áreas de dentina exposta, indicado quando todos os outros métodos de alívio da dor falham ou quando há questões estéticas envolvidas ${ }^{2,22}$.
Verifica-se que embora vários tratamentos tenham sido propostos, ainda não se estabeleceu o agente ideal para solucionar essa patologia que possui um grande potencial de recidiva ${ }^{8}$.

\section{Discussão}

Atualmente, a Teoria Hidrodinâmica é aceita como apropriada para suportar os mecanismos subjacentes responsáveis pela HSDC. Nessa teoria, demonstrou-se que há um fluxo normal de fluidos através da dentina, assim como ocorrem estímulos apropriados à taxa de escoamento do fluido que aumenta e empurra os nervos para dentro da dentina pulpar $^{23,24}$.

A hipersensibilidade dentinária acontece quando os túbulos dentinários estão expostos, porém, a exposição pode dar-se com ou sem perda visível de estrutura dental. A multifatoriedade de sua etiologia pode ser uma das causas do fracasso de seu tratamento ${ }^{4,5,18,19}$.

Ao se deparar com um caso de hipersensibilidade um diagnóstico diferencial se faz necessário, pois existem outras patologias com sintomas semelhantes aos da HSDC, mas que requerem tratamentos diferentes. Assim, cada tipo de lesão dentinária tem características clínicas próprias e procedimentos de controle diferentes a serem associados ao tratamento $0^{12,18-20}$.

Em lesões de abrasão, a atenção deve ser enfatizada para as orientações da técnica e da pressão utilizada na escovação, bem como para os tipos de escovas e de dentifrícios usados ${ }^{28,30}$. Apesar de não ser consenso, há autores que afirmam que a escova elétrica causa causa menor desgaste, pois realiza mínimna pressão na escovação. ${ }^{29} \mathrm{Em}$ lesões por abfração, os procedimentos de controle e de prevenção são mais complexos, envolvendo ajustes oclusais e restaurações ${ }^{3}$.

Para o tratamento da hipersensibilidade dentinária, as indicações recaem desde métodos que $o$ próprio paciente realiza em casa, como os dentifrícios dessensibilizantes até a possibilidade de utilização dos lasers ${ }^{2,3,16,23,25,26,30-34}$. O fluoreto de sódio está indicado como tratamento, mas não foi, ainda, confirmado que o fechamento dos túbulos dê-se em apenas uma sessão. Portanto, quando aplicado na superfície dentinária exposta, a indicação é de que o procedimento seja realizado por quatro minutos, uma vez por semana, durante quatro semanas, diminuindo, assim, a sensibilidade dos dentes tratados ${ }^{13,15}$.

$\mathrm{O}$ tratamento com laser tem demonstrado bastante eficiência na dessensibilização de dentina, porém, ainda significa custo alto para o paciente e para o profissional. E as técnicas cirúrgicas para correção da retração gengival são mais utilizadas quando há também um envolvimento estético ${ }^{22}$.

A escolha por um tratamento ideal precisa, necessariamente, ser avaliada de forma individualizada. Alguns pacientes são relutantes ao controle de dietas alimentares, outros se adaptam ao controle 
de dieta, mas não são capazes de melhorar a higiene oral. Nenhum tratamento possui todas as vantagens que precisamos, mas da mesma forma, poucos pacientes conseguem uma adaptação perfeita ao tratamento indicado e isso precisa ser ponderado para o êxito da terapia proposta ${ }^{22}$.

Mudança de hábitos, como evitar uma escovação com técnica inadequada, evitar a utilização de escova dental com cerdas duras e evitar dentifrícios com alta abrasividade trazem grandes benefícios aos pacientes em longo prazo. Conselhos alimentares, medidas de estimulação do fluxo salivar, aplicação tópica de fluoretos, monitoramento e adequação de técnicas de escovação com dentifrícios dessensibilizantes com baixa abrasividade e alto teor de flúor são medidas que sem dúvida podem reduzir e prevenir a hipersensibilidade dentinária ${ }^{35}$.

O diagnóstico clínico precoce ainda é a melhor maneira de se prevenir, acompanhada, indiscutivelmente, de uma anamnese completa seguida de um exame detalhado.

\section{Considerações finais}

A teoria mais aceita para explicar o mecanismo que provoca a dor na hipersensibilidade dentinária é a Teoria Hidrodinâmica. Dessa forma, conhecer a etiologia da hipersensibilidade dentinária é importante para se alcançar a obliteração dos túbulos dentinários, resultando na efetiva redução do movimento do fluido e na diminuição da dor.

Os fatores etiológicos encontrados na literatura sugerem desde uma escovação exagerada, com dentifrícios abrasivos, de uma dieta rica em alimentos e bebidas ácidas até o estresse oclusal, entre outros.

Todos os tratamentos apresentados são eficazes na redução da hipersensibilidade dentinária, porém, apresentam efeitos diferentes. $\mathrm{O}$ tratamento restaurador é um procedimento eficiente e duradouro. Os agentes dessensibilizantes, do tipo materiais adesivos, oxalato férrico, oxalato de potássio, nitrato de potássio, soluções de fosfato de cálcio, fluoretos, vernizes fluorados, possuem na literatura eficiência indiscutível. As terapias a laser que iniciaram tão singelas, atualmente, mostram-se cada vez mais indicadas com resultados e estudos promissores.

No entanto, os estudos que mostram prevalência ainda são escassos e com delineamento metodológico inadequado, portanto é necessária a realização de estudos epidemiológicos com amostras representativas para se conhecer a prevalência e os fatores associados a esse quadro na população brasileira.

\section{Abstract}

Introduction: the dentin hypersensitivity (HSDC) is characterized by loss of enamel and cementum in the cervical region of the teeth and the consequent exposure of dentinal tubules leading to a painful and uncomfortable for the patient. Objective: This study aimed to address the etiological factors, prevalence and various forms related to cervical dentin hypersensitivity treatment. Literature review: Being reported with a prevalence of about $30 \%$ of the population, this loss of tooth structure occurs due to the formation of cervical lesions by processes of gingival recession, abrasion, erosion, abfraction or association of two or more factors. The knowledge of the etiology of any disease or oral condition is paramount for safe and effective treatment, so this literature review is to inform the etiology, prevalence, and treatment options for dentin hypersensitivity, through a literature review of national and international data-bases (pubmed, scielo, bireme and cochrane). Some medical treatments have to be effective, such as application of potassium oxalate, strontium chloride, fluorinated coatings, sodium fluoride, low power laser, desensitizing dentifrice systems, adhesives and restorative procedures. Final considerations: Therefore, the identification and removal of etiological factors are essential to the successful treatment of HSDC when associated with obliteration of the dentinal tubules, resulting in the effective reduction of the movement of the fluid within the tubules and decreased pain.

Keywords: Diagnosis. Dentin sensitivity. Prevention \& control. Hydrodynamics.

\section{Referências}

1. Kimura Y, Wilder-Smith P, Yonaga K, Matsumoto K. Treatment of dentine hypersensitivity by lasers: a review. J Clin Periodontol 2000; 27(10):715-21.

2. Aranha AC. Hipersensibilidade dentinária e lesões cervicais não cariosas: causas, etiologia e prevalência. Rev $\mathrm{ABO}$ Nacional 2009; 17(4):5-9.

3. Sobral MAP, Netto NG. Aspectos clínicos da etiologia da hipersensibilidade dentinária cervical. Rev Odontol Univ São Paulo 1999; 13(2):189-95.

4. Aw TC, Lepe X, Jonhson GH, Mancl L. Characteristics of non carious cervical lesions: a clinical investigation. J Am Dent Assoc 2002; 133(6):725-33.

5. Tonetto MR Dantas AAR, Bortolin GF, Fabris M, de Campos E A, Andrade MF. Hipersensibilidade dentinária cervical: em busca de um tratamento eficaz. Rev Odontol Univ São Paulo 2012; 24(3):190-9.

6. Hara AT, Kelly AS, Gonzaléz-CC, Eckert CJ, Barlow AP, Mason SC,et al. Influence of dentifrices on eroded enamel remineralized in situ. Caries Res 2009; 43(1):57-63.

7. Turssi CP. Abrasão dentinária: papel de aspectos relacionados ao dentifrício, escova dental e as práticas de escovação. Rev ABO Nacional 2009; 17(4):16-21.

8. Barbosa RPS, Santos RL, Gusmão ES. Terapias para controle das lesões não cariosas hipersensíveis. Odontologia Clin Científica 2005; 4(93):171-6. 
9. Addy M, West NX. The role of toothpaste in the aethiology and treatment of dentine hypersensitive. Monog Oral Sci. 2013; 23:75-87.

10. Matias MNA, Leão JC, Filho PFM, Silva CHV. Hipersensibilidade dentinária: uma revisão da literatura. Odonto Clin Cient (online) 2010; 9 (3):205-8.

11. Liu HC, Lan WH, Hsieh CC. Prevalence and distribuition of cervical dentin hypersensitivity in a population in Taipei, Taiwan. J Endod 1998; 24(1):45-7.

12. Palma ABO, Costa SM, Resende VLS, Neves AD, Abreu MHNG, Guedes CAS, et al. Prevalência da Hipersensibilidade dentinária cervical nos pacientes da Clínica Integrada I da Unimontes - Montes Claros/MG. Pesq Bras Odontoped Clin Integr 2005; 5(1):29-34.

13. Pinto SCS, Pochapski MT, Wambier DS, Pilatti GL, Santos FA. Análise de substâncias dessensibilizantes na permeabilidade da dentina e obliteração de túbulos dentinários: estudo in vitro. Period 2007; 17(3):41-8.

14. Vianna RBC. Abrasão em relação ao desgaste dentário e consequente hipersensibilidade da dentina. Rev ABO Nac 2009; 17(4):1-4.

15. Pereira JC, Martineli ACB, Figueiredo S, Sérgio L. Tratamento da hipersensibilidade dentinária com três diferentes formulações à base de oxalato de potássio: estudo clínico. Fac Odontol Bauru 2001; 9(3/4):123-30.

16. Garcia G, Rossel FL, Sampaio JEC. Tratamento da hipersensibilidade dentinária. RGO 1996; 44 (4):237-9.

17. Tamaro S, Wennstron JL, Bergonholtz O. Root dentin sensitivity following non cirurgical periodontal treatment. J Clin Periodontol 2000; 27(9):690-97.

18. Ganss G, Lussi A. Diagnosis of tooth wear. Monogr Oral Sci $2006 ; 20: 32-43$

19. Lussi A. Erosive tooth wear: a multifactorial condition of growing concern and increasing knowledge. Monogr Oral Sci 2006; 20:1-8.

20. Corso S, Padilha DMP, Corso AC, Hugo FN. Avaliação do potencial erosivo de sucos de frutas artificiais em pó, refrigerantes, isotônicos e chás enlatados disponíveis comercialmente no Brasil. Rev Fac Odontol Univ Passo Fundo 2006; 11(1):45-50.

21. Linfante-Oliva C, López Jornet P, Camacho Alonso F, EsteveSalinas J. Study of oral changes in patients with eating disorders. Int J Dent Hyg 2008; 6(2):119-22.

22. Tinoco EMB. Relevância clínica da abrasão: implicações e recomendações para os pacientes. Rev ABO Nac 2009; 17(4):22-4.

23. Brännström M. The effect of dentin desiccation and aspirated odontoblasts on the pulp. Oper Dent 1967; 20(2):165-71.

24. Pashley DH. Theory of dentin sensitivity. J Clin Dent 1994 spec. issue - (5):65-7.

25. Assis CA, Antoniazzo RP, Zanatta FB, Rösing CK. Efficacy of gluma desensitizer on dentin hypersensitivity in periodontally treated patients. Braz Oral Res 2006; 20(3): 252-6.

26. Mehmood Z, Shah JA, Javed MU, Manzoor MA, Asghar I, Saeed MH. Efficacy of gluma desensitizer TM and duraphat TM in relieving dentinal hypersensivity in non-carious cervical lesions. PODJ 2011; 31(1):183-6.

27. Pandit N, Gupta R, Bansal A. Comparative evaluation of two commercially available desensitizing agents for the treatment of dentinal hypersensitivity. Indian J Dent Res 2012; 23(6):778-83.

28. Prabhakar AR, Manojkumar AJ, Basappa N. In vitro remineralization of enamel subsurface lesions and assessment of dentine tubule occlusion from $\mathrm{NaF}$ dentifrices with and without calcium. J Indian Soc Pedo Prev Dent 2013; 31(1):29-35.
29. Orsini G, Procaccini M, Manzoli L, Sparabombe S, Tiriduzzi $\mathrm{P}$, Bambini $\mathrm{F}$ et al. A 3-Day randomized clinical trial to investigate the desensitizing properties of three dentifrices. J Periodontol 2013; 1(2):184-92.

30. Umana M, Heysselaer D, Tielemans M, Compere P, Zeinoun T, Nammour S. Dentinal tubules sealing by means of diode lasers (810 and $980 \mathrm{~nm}$ ): a preliminary in vitro study. Photomed Laser Surg 2013; 31(7):307-14.

31. Shintome LK, Umetsubo LS, Nagayassu MP, Jorge ALC, Gonçalves SE de P, Torres CRG. Avaliação clínica da laserterapia no tratamento da hipersensibilidade dentinária. Cienc Odontol Bras 2007; 10(1):26-33.

32. Verma SK, Maheshwari S, Singh RK, Chaudhari PK. Laser in dentistry: An innovative tool in modern dental practice. Nat J Maxillofac Surg 2012; 3(2):124-32.

33. Han SY, Jung HI, Kwon HK, Kim BI. Combined effects of Er:YAG laser and nano-carbonate apatite dentifrice on dentinal tubule occlusion: in vitro study. Photomed Laser Surg 2013; 31(7):342-8.

34. Lopes AO, Aranha AC. Comparative evaluation of the effects of Nd: YAG laser and a desensitizer agent on the treatment of dentin hypersensitivity: a clinical study. Photomed Laser Surg 2013; 31(3):132-8.

35. Wiegand A, Meier W, Sutter E, Magalhães AC, Becker K, Roos $\mathrm{M}$ et al. Protective effect of different tetrafluorides on erosion of pellicle-free and pellicle-covered enamel and dentine. Caries Res 2008; 42(4):247-54.

\section{Endereço para correspondência:}

Micheline Sandini Trentin

Rua Bento Gonçalves, 651/1301

99010-010 Passo Fundo-RS

Fone: (54) 3316-8402

E-mail: tmicheline@upf.br

Recebido: 15/10/2013. Aceito: 07/08/2014. 